Annals of Pure and Applied Mathematics

Vol. 14, No. 1, 2017, 173-181

ISSN: 2279-087X (P), 2279-0888(online)

Published on 9 August 2017

Annals of

www.researchmathsci.org

DOI: http://dx.doi.org/10.22457/apam.v14n1a20

Pure and Applied

Mathematics

\title{
Multiplicative Operations of Intuitionistic Fuzzy Matrices
}

\author{
D.Venkatesan $^{1}$ and S.Sriram ${ }^{2}$ \\ ${ }^{1}$ Department of Mathematics \\ Annamalai University, Annamalainagar -608002, India \\ Email: venkat2733327@gmail.com \\ ${ }^{2}$ Mathematics Wing, Directorate of Distance Education \\ Annamalai University, Annamalainagar -608002, India \\ Corresponding author. Email: ssm_3096@yahoo.co.in
}

Received 28 June 2017; accepted 26 July 2017

\begin{abstract}
In this paper, we investigate the algebraic properties of intuitionistic fuzzy matrices under the new operations $X_{1}$ and $X_{2}$. The properties of intuitionistic fuzzy matrices are also obtained in the case where these new operations are combined with the well known operations of intuitionistic fuzzy matrices.
\end{abstract}

Keywords: Intuitionistic fuzzy set, intuitionistic fuzzy matrix

AMS Mathematics Subject Classification (2010): 94D05, 15B15, 15B99

\section{Introduction}

Atanassov [2] introduced the concept of intuitionistic fuzzy set (IFS) which is the generalization of fuzzy set introduced by Zadeh [22]. Since its appearance, intuitionistic fuzzy set has been investigated by many researchers and applied to many fields, such as decision making, clustering analysis etc.,

Using the fuzzy sets Kim and Roush [9] studied fuzzy matrices as a generalization of matrices over the two element Boolean algebra. Shyamal and Pal [19] studied the two new operators on fuzzy matrices and their algebraic properties. In Zhang and Zheng [23] defined two fuzzy operators and four cut sets for fuzzy matrices and studied their algebraic properties. Using the theory of intuitionistic fuzzy set, Im et al. [6] defined the notion of intuitionistic fuzzy matrix (IFM) as a generalization of fuzzy matrix.

IFM is very useful in the discustion of intuitionistic fuzzy relation(IFR) [5, 2 2]. Lee and Jeong [11] obtained a canonical form of the transtive IFM. Sriram and Murugadas [16] proved the set of all IFMs form a semiring with respect to max-min composition of IFMs. Zhong et al. [21] constraucted the intuitionistic fuzzy similarity matrix and then utilize it to derive a method for clustering analysis.

Simultaneously, Pal et al. [8] defined the IFM and Pal [15] introduced the intuitionistic fuzzy Determinant, studied some properties on it. Khan and Pal [9] studied some operations on IFMs. Shyamal and Pal [18] studied the distances between intuitionistic fuzzy matrices and their algebraic properties. Adak et al. [1] defined the concept of intuitionistic fuzzy block matrices and different types of intuitionistic fuzzy 


\section{D.Venkatesan and S.Sriram}

block matrices. Also, the operations direct sum, Kronecker sum, Kronecker product of intuitionistic fuzzy matrices are presented.

Boobalan and Sriram $[4,17]$ studied the algebraic sum and algebraic product of two intuitionistic fuzzy matrices and their algebraic properties. Also they proved the set of all intuitionistic fuzzy matrices forms a commutative monoid with respect to these operations. Muthuraji et al. [13] introduced a new composition operator and studied the algebraic properties also obtained a decomposition of an IFM. Muthuraji and Sriram [14] defined two operators namely Lukasiwicz disjunction and conjunction and investigated its algebraic properties also results which connects the above set operators with the other existing operators.

Emam and Fndh [6] defined some kinds of IFMs, the max-min and min-max composition of IFMs. Also they derived several importent results by these compositions and constract an idempotent intuitionistic fuzzy matrix from any given one through the min-max composition.

Recently, Pal $[24,25]$ defines new kind of fuzzy matrices. In these matrices rows and columns are also uncertain.

In [3] Atanassov, five new intuitionistic fuzzy operations on intuitionistic fuzzy sets containing multiplication were introduced and their properties are studied.

In this paper, we extend two of these operations to IFMs and investigate their algebraic properties.

\section{Preliminaries}

In this section, we give to some basic definitions of intuitionistic fuzzy matrix that are necessary for this paper.

Definition 2.1. ([7]) An intuitionistic fuzzy matrix(IFM) is a matrix of pairs $A=\left(\left\langle a_{i j}, a_{i j}^{\prime}\right\rangle\right)$ of a non negative real numbers satisfying $0 \leq a_{i j}+a_{i j}^{\prime} \leq 1$ for all $\mathrm{i}, \mathrm{j}$.

Definition 2.2. ([15]) For any two IFMs $A$ and $B$ of same size, we have

(i) The max-min composition of $A$ and $B$ is defined by

$A \vee B=\left(\left\langle\max \left(a_{i j}, b_{i j}\right), \min \left(a_{i j}^{\prime}, b_{i j}^{\prime}\right)\right\rangle\right)$.

(ii) The min-max composition of $A$ and $B$ is defined by

$A \wedge B=\left(\left\langle\min \left(a_{i j}, b_{i j}\right), \max \left(a_{i j}^{\prime}, b_{i j}^{\prime}\right)\right\rangle\right)$.

Definition 2.3. ([15]) For any two IFMs $A$ and $B$ of same size, $A \geq B$ iff $a_{i j} \geq b_{i j}$ and $a_{i j}^{\prime} \leq b_{i j}^{\prime}$ for all $\mathrm{i}, \mathrm{j}$.

Definition 2.4. ([4]) The $m \times n$ zero IFM $O$ is an IFM all of whose entries are $\langle 0,1\rangle$. The $m \times n$ universal IFM $J$ is an IFM all of whose entries are $\langle 1,0\rangle$.

Definition 2.5. ([15]) The complement of an IFM $A$ which is denoted by $A^{C}$ and is defined by $A^{C}=\left(\left\langle a_{i j}^{\prime} \cdot a_{i j}\right\rangle\right)$.

Lemma 2.6. ([21]) Let $a, b$ and $c$ be real numbers. The the following equalities hold: 
Multiplicative Operations of Intuitionistic Fuzzy Matrices

(i) $\max (a, \min (a, b)=a, \min (a, \max (a, b))=a$.

(ii) $\max (a, \max (b, c))=\max (\max (a, b), c), \min (a, \min (b, c)=\min (\min (a, b), c)$.

Lemma 2.6. ([21]) Let $a, b \in[0,1]$, we have

(i) $\max (a, \min (b, c))=\min (\max (a, b), \max (a, c))$,

$\min (a, \max (b, c))=\max (\min (a, b), \min (a, c))$.

(ii) $\max (a, b) \max (a, c) \leq \max (a, b c), \min (a, b) \min (a, c) \geq \min (a, b c)$.

3. Main results

In this section, we difine operations $X_{1}$ and $X_{2}$ of IFMs and investigate their algebraic properties.

Definition 3.1. For any two IFMs $A$ and $B$ of same size, then we define

(i) $A X_{1} B=\left(\left\langle\max \left(a_{i j}, b_{i j}\right), a_{i j}^{\prime} b_{i j}^{\prime}\right\rangle\right)$.

(ii) $A X_{2} B=\left(\left\langle a_{i j} b_{i j}, \max \left(a_{i j}^{\prime}, b_{i j}^{\prime}\right)\right\rangle\right)$.

$a_{i j} b_{i j}$ and $a_{i j}^{\prime} b_{i j}^{\prime}$ are the ordinary multiplications.

Property 3.2. If $A$ and $B$ are any two IFMs of same size, we have $A X_{2} B \leq A X_{1} B$.

Proof: Let $A=\left(\left\langle a_{i j}, a_{i j}^{\prime}\right\rangle\right)$ and $B=\left(\left\langle b_{i j}, b_{i j}^{\prime}\right\rangle\right)$ be two IFMs of same size.

Since $a_{i j} b_{i j} \leq \min \left(a_{i j}, b_{i j}\right) \leq \max \left(a_{i j}, b_{i j}\right)$ and $\max \left(a_{i j}^{\prime}, b_{i j}^{\prime}\right) \geq a_{i j}^{\prime} b_{i j}^{\prime}$, for all $\mathrm{i}, \mathrm{j}$.

Hence, $A X_{2} B \leq A X_{1} B$.

Property 3.3. For any IFM $A$, we have

(i) $A X_{1} A \neq A$.

(ii) $A X_{2} A \neq A$.

Proof: Let $A=\left(\left\langle a_{i j}, a_{i j}^{\prime}\right\rangle\right)$ be an IFM. Then

(i) $A X_{1} A=\left(\left\langle a_{i j}, a_{i j}^{\prime 2}\right\rangle\right)$

$$
\neq\left(\left\langle a_{i j}, a_{i j}^{\prime}\right\rangle\right)
$$

Hence, $A X_{1} A \neq A$.

$$
\text { (ii) } \begin{aligned}
A X_{2} A & =\left(\left\langle a_{i j}^{2}, a_{i j}^{\prime}\right\rangle\right) \\
& \neq\left(\left\langle a_{i j}, a_{i j}^{\prime}\right\rangle\right) .
\end{aligned}
$$

Hence, $A X_{2} A \neq A$.

The following properties are obvious. The operations $X_{1}$ and $X_{2}$ are commutative as well as associative.

Property 3.4. Let $A, B$ and $C$ be any three IFMs of same size, we have

(i) $A X_{1} B=B X_{1} A$.

(ii) $\left(A X_{1} B\right) X_{1} C=A X_{1}\left(B X_{1} C\right)$.

(iii) $A X_{2} B=B X_{2} A$.

(iv) $\left(A X_{2} B\right) X_{2} C=A X_{2}\left(B X_{2} C\right)$.

Property 3.5. For any three IFMs $A, B$ and $C$ of same size, we have

(i) Nullity: $A X_{1} J=J, A X_{2} O=O$.

(ii) Identity: $A X_{1} O=A, A X_{2} J=A$. 
D.Venkatesan and S.Sriram

(iii) Distributivity: $A X_{1}\left(B X_{2} C\right) \neq\left(A X_{1} B\right) X_{2}\left(A X_{1} C\right)$ and

$$
A X_{2}\left(B X_{1} C\right) \neq\left(A X_{2} B\right) X_{1}\left(A X_{2} C\right) \text {. }
$$

(iv) Absorption: $A X_{1}\left(A X_{2} B\right) \neq A$ and $A X_{2}\left(A X_{1} B\right) \neq A$.

Proof: $(i)$ and (ii) are clear by the definition of $X_{1}$ and $X_{2}$.

(iii) $A X_{1}\left(B X_{2} C\right)=\left(\left\langle\max \left(a_{i j}, b_{i j} c_{i j}\right), a_{i j}^{\prime} \max \left(b_{i j}^{\prime}, c_{i j}^{\prime}\right)\right\rangle\right)$

$\left(A X_{1} B\right) X_{2}\left(A X_{1} C\right)=\left(\left\langle\max \left(a_{i j}, b_{i j}\right) \max \left(a_{i j}, c_{i j}\right), \max \left(a_{i j}^{\prime} b_{i j}^{\prime}, a_{i j}^{\prime} c_{i j}^{\prime}\right)\right\rangle\right)$

$=\left(\left\langle\max \left(a_{i j}, b_{i j}\right) \max \left(a_{i j}, c_{i j}\right), a_{i j}^{\prime} \max \left(b_{i j}^{\prime}, c_{i j}^{\prime}\right)\right\rangle\right)$

Since max $\left(a_{i j}, b_{i j}\right) \max \left(a_{i j}, c_{i j}\right) \leq \max \left(a_{i j}, b_{i j} c_{i j}\right)$, for all $\mathrm{i}, \mathrm{j}$.

Hence, $A X_{1}\left(B X_{2} C\right) \neq\left(A X_{1} B\right) X_{2}\left(A X_{1} C\right)$.

Similarly we can prove the other one.

(iv) $A X_{1}\left(A X_{2} B\right)=\left(\left\langle\max \left(a_{i j}, a_{i j} b_{i j}\right), a_{i j}^{\prime} \max \left(a_{i j}^{\prime}, b_{i j}^{\prime}\right)\right\rangle\right)$

$=\left(\left\langle a_{i j}, a_{i j}^{\prime} \max \left(a_{i j}^{\prime}, b_{i j}^{\prime}\right)\right\rangle\right)$

Hence, $A X_{1}\left(A X_{2} B\right) \neq A$.

$$
\neq\left(\left\langle a_{i j}, a_{i j}^{\prime}\right\rangle\right) \text {. }
$$

Similarly we can prove the other one.

\section{Results on complement of IFM}

The operator complement obey the De Morgan's laws for the operations $X_{1}$ and $X_{2}$.

This is established in the following properties.

Property 4.1. For the IFMs $A$ and $B$ of same size, we have

(i) $\left(A X_{1} B\right)^{C}=A^{C} X_{2} B^{C}$.

(ii) $\left(A X_{2} B\right)^{C}=A^{C} X_{1} B^{C}$.

(iii) $\left(A X_{1} B\right)^{C} \leq A^{C} X_{1} B^{C}$.

(iv) $\left(A X_{2} B\right)^{C} \leq A^{C} X_{2} B^{C}$.

Proof: $(i)\left(A X_{I} B\right)^{C}=\left(\left\langle a_{i j}^{\prime} b_{i j}^{\prime}, \max \left(a_{i j}, b_{i j}\right)\right\rangle\right)$

Hence, $\left(A X_{1} B\right)^{C}=A^{C} X_{2} B^{C}$.

$$
=A^{C} X_{2} B^{C} \text {. }
$$

(ii) $\left(A X_{2} B\right)^{C}=\left(\left\langle\max \left(a_{i j}^{\prime}, b_{i j}^{\prime}\right), a_{i j} b_{i j}\right\rangle\right)$

$$
=A^{C} X_{1} B^{C} \text {. }
$$

Hence, $\left(A X_{2} B\right)^{C}=A^{C} X_{1} B^{C}$.

(iii) Since $a_{i j}^{\prime} b_{i j}^{\prime} \leq \max \left(a_{i j}^{\prime}, b_{i j}^{\prime}\right), \max \left(a_{i j}, b_{i j}\right) \geq a_{i j} b_{i j}$ and from the Definition (2.3) $\left(A X_{1} B\right)^{C} \leq A^{C} X_{1} B^{C}$.

The proof (iv) is similar to that of (iii).

Property 4.2. For any IFM $A$, we have

(i) $A X_{1} A^{C} \neq 0$.

(ii) $A X_{2} A^{C} \neq J$.

Proof: (i) $A X_{1} A^{C}=\left(\left\langle\max \left(a_{i j}, a_{i j}^{\prime}\right), a_{i j}^{\prime} a_{i j}\right\rangle\right)$

Hence, $A X_{1} A^{C} \neq O$.

$$
\neq\langle 0,1\rangle \text {. }
$$

(ii) $A X_{2} A^{C}=\left(\left\langle a_{i j} a_{i j}^{\prime}, \max \left(a_{i j}, a_{i j}^{\prime}\right)\right\rangle\right)$ 
Multiplicative Operations of Intuitionistic Fuzzy Matrices

$$
=\langle 1,0\rangle \text {. }
$$

Hence, $A X_{2} A^{C} \neq J$.

Property 4.3. For any IFM $A$, we have

(i) $\left(A X_{1} A^{C}\right)^{C}=A X_{2} A^{C}$.

(ii) $\left(A X_{2} A^{C}\right)^{C}=A X_{1} A^{C}$.

Proof: $A X_{1} A^{C}=\left(\left\langle\max \left(a_{i j}, a_{i j}^{\prime}\right), a_{i j}^{\prime} a_{i j}\right\rangle\right)$ and $A X_{2} A^{C}=\left(\left\langle a_{i j} a_{i j}^{\prime}, \max \left(a_{i j}, a_{i j}^{\prime}\right)\right\rangle\right)$.

(i) $\left(A X_{1} A^{C}\right)^{C}=\left(\left\langle a_{i j}^{\prime} a_{i j}, \max \left(a_{i j}, a_{i j}^{\prime}\right)\right\rangle\right)$

$$
\begin{aligned}
& =\left(\left\langle a_{i j} a_{i j}^{\prime}, \max \left(a_{i j}^{\prime}, a_{i j}\right)\right\rangle\right) \\
& =A X_{2} A^{C} .
\end{aligned}
$$

Hence, $\left(A X_{1} A^{C}\right)^{C}=A X_{2} A^{C}$.

(ii) $\left(A X_{2} A^{C}\right)^{C}=\left(\left\langle\max \left(a_{i j}^{\prime}, a_{i j}\right), a_{i j} a_{i j}^{\prime}\right\rangle\right)$

$$
\begin{aligned}
& =\left(\left\langle\max \left(a_{i j}, a_{i j}^{\prime}\right), a_{i j}^{\prime} a_{i j}\right\rangle\right) \\
& =A X_{1} A^{C} .
\end{aligned}
$$

Hence, $\left(A X_{2} A^{C}\right)^{C}=A X_{1} A^{C}$.

Property 4.4. For any two IFMs $A$ and $B$ of same size, we have

(i) $\left(A X_{1} A^{C}\right) X_{1}\left(A X_{1} A^{C}\right) \neq A X_{1} A^{C}$.

(ii) $\left(A X_{2} A^{C}\right) X_{2}\left(A X_{2} A^{C}\right) \neq A X_{2} A^{C}$.

Proof: $(i)\left(A X_{l} A^{C}\right) X_{l}\left(A X_{l} A^{C}\right)=\left(\left\langle\max \left(\max \left(a_{i j}, a_{i j}^{\prime}\right), \max \left(a_{i j}, a_{i j}^{\prime}\right)\right),\left(a_{i j}, a_{i j}^{\prime}\right)^{2}\right\rangle\right)$.

$A X_{1} A^{C}=\left(\left\langle\max \left(a_{i j}, a_{i j}^{\prime}\right), a_{i j}^{\prime} a_{i j}\right\rangle\right)$.

Since $\left(a_{i j}, a_{i j}^{\prime}\right)^{2} \leq a_{i j}^{\prime} a_{i j}$, for all $\mathrm{i}, \mathrm{j}$.

Hence, $\left(A X_{1} A^{C}\right) X_{1}\left(A X_{1} A^{C}\right) \neq A X_{1} A^{C}$.

The proof $(i i)$ is similar to that of $(i)$.

\section{Results on $X_{1}$ and $X_{2}$ combined with max-min and min-max compositions of} IFMs

We shall discuss the absorption property in the case where the operations $X_{1}, X_{2}$, max-min and min-max compositions are combined each other.

Property 5.1. Let $A$ and $B$ are any two IFMs of same size, we have

(i) $A X_{l}(A \vee B) \neq A$.

(ii) $A X_{l}(A \wedge B) \neq A$.

Proof: $(i) A X_{I}(A \vee B)=\left(\left\langle\max \left(a_{i j}, \max \left(a_{i j}, b_{i j}\right), a_{i j}^{\prime} \min \left(a_{i j}^{\prime}, b_{i j}^{\prime}\right)\right\rangle\right)\right.$

$$
\begin{aligned}
& =\left(\left\langle a_{i j}, a_{i j}^{\prime} \min \left(a_{i j}^{\prime}, b_{i j}^{\prime}\right)\right\rangle\right) \\
& \neq\left(\left\langle a_{i j}, a_{i j}^{\prime}\right\rangle\right) .
\end{aligned}
$$

Hence, $A X_{1}(A \vee B) \neq A$.

The proof $(i i)$ is similar to that of $(i)$.

Property 5.2. Let $A$ and $B$ are any two IFMs of same size, we have

(i) $A X_{2}(A \vee B) \neq A$.

(ii) $A X_{2}(A \wedge B) \neq A$.

Proof: $(i) A X_{2}(A \vee B)=\left(\left\langle a_{i j} \max \left(a_{i j}, b_{i j}\right), \max \left(a_{i j}^{\prime} \min \left(a_{i j}^{\prime}, b_{i j}^{\prime}\right)\right)\right\rangle\right)$ 
Hence, $A X_{2}(A \vee B) \neq A$.

$$
\begin{aligned}
& =\left(\left\langle a_{i j} \max \left(a_{i j}, b_{i j}\right), a_{i j}^{\prime}\right\rangle\right) \\
& \neq\left(\left\langle a_{i j}, a_{i j}^{\prime}\right\rangle\right) .
\end{aligned}
$$

The proof $(i i)$ is similar to that of $(i)$.

Property 5.3. Let $A$ and $B$ are any two IFMs of same size, we have

(i) $A \vee\left(A X_{1} B\right) \neq A X_{1} B$.

(ii) $A \wedge\left(A X_{1} B\right)=A$.

Proof: $(i) A \vee\left(A X_{I} B\right)=\left(\left\langle\max \left(a_{i j}, \max \left(a_{i j}, b_{i j}\right)\right), \min \left(a_{i j}^{\prime}, a_{i j}^{\prime} b_{i j}^{\prime}\right)\right\rangle\right)$

$$
\begin{aligned}
& =\left(\left\langle\max \left(a_{i j}, \max \left(a_{i j}, b_{i j}\right)\right), a_{i j}^{\prime} b_{i j}^{\prime}\right\rangle\right) \\
& \neq A X_{1} B .
\end{aligned}
$$

Hence, $A \vee\left(A X_{1} B\right) \neq A X_{1} B$.

(ii) $A \wedge\left(A X_{1} B\right)=\left(\left\langle\min \left(a_{i j}, \max \left(a_{i j}, b_{i j}\right)\right), \max \left(a_{i j}^{\prime}, a_{i j}^{\prime} b_{i j}^{\prime}\right)\right\rangle\right)$

$$
=\left(\left\langle a_{i j}, a_{i j}^{\prime}\right\rangle\right) \text {. }
$$

Hence, $A \wedge\left(A X_{l} B\right)=A$.

Similarly, we can prove the following property

Property 5.4. Let $A$ and $B$ are any two IFMs of same size, we have

(i) $A \wedge\left(A X_{2} B\right) \neq A X_{2} B$.

(ii) $A \vee\left(A X_{2} B\right)=A$.

Next, we shall discuss the distributivity in the case where the operations $X_{1}, X_{2}$, max-min and min-max compositions are combined each other.

Property 5.5. Let $A, B$ and $C$ are any three IFMs of same size, we have

(i) $A X_{l}(B \vee C)=\left(A X_{l} B\right) \vee\left(A X_{l} C\right)$.

(ii) $A X_{1}(B \wedge C)=\left(A X_{1} B\right) \wedge\left(A X_{1} C\right)$.

Proof: $(i) A X_{I}(B \vee C)=\left(\left\langle\max \left(a_{i j},\left(\max \left(b_{i j}, c_{i j}\right)\right), a_{i j}^{\prime} \min \left(b_{i j}^{\prime}, c_{i j}^{\prime}\right)\right\rangle\right)\right.$

$$
\begin{aligned}
& =\left(\left\langle\max \left(\max \left(a_{i j}, b_{i j}\right), \max \left(a_{i j}, c_{i j}\right), \min \left(a_{i j}^{\prime} b_{i j}^{\prime}, a_{i j}^{\prime} c_{i j}^{\prime}\right)\right\rangle\right)\right. \\
& =\left(A X_{I} B\right) \vee\left(A X_{I} C\right) .
\end{aligned}
$$

Hence, $A X_{1}(B \vee C)=\left(A X_{1} B\right) \vee\left(A X_{1} C\right)$.

The proof $(i i)$ is similar to that of $(i)$.

Property 5.6. Let $A, B$ and $C$ are any three IFMs of same size, we have

(i) $A X_{2}(B \vee C)=\left(A X_{2} B\right) \vee\left(A X_{2} C\right)$.

(ii) $A X_{2}(B \wedge C)=\left(A X_{2} B\right) \wedge\left(A X_{2} C\right)$.

Proof: $(i) A X_{2}(B \vee C)=\left(\left\langle a_{i j}\left(\max \left(b_{i j}, c_{i j}\right), \max \left(a_{i j}^{\prime} \min \left(b_{i j}^{\prime}, c_{i j}^{\prime}\right)\right\rangle\right)\right.\right.$

$$
\begin{aligned}
& =\left(\left\langle\max \left(a_{i j} b_{i j}, a_{i j} c_{i j}\right), \min \left(\max \left(a_{i j}^{\prime}, b_{i j}^{\prime}\right), \max \left(a_{i j}^{\prime}, c_{i j}^{\prime}\right)\right\rangle\right)\right. \\
& =\left(A X_{2} B\right) \vee\left(A X_{2} C\right) .
\end{aligned}
$$

Hence, $A X_{2}(B \vee C)=\left(A X_{2} B\right) \vee\left(A X_{2} C\right)$.

The proof $(i i)$ is similar to that of $(i)$.

Property 5.7. Let $A, B$ and $C$ are any three IFMs of same size, we have 
Multiplicative Operations of Intuitionistic Fuzzy Matrices

(i) $A \vee\left(B X_{1} C\right) \neq(A \vee B) X_{l}(A \vee C)$

(ii) $A \wedge\left(B X_{l} C\right) \neq(A \wedge B) X_{l}(A \wedge C)$.

Proof: $(i) A \vee\left(B X_{1} C\right)=\left(\left\langle\max \left(a_{i j}, \max \left(b_{i j}, c_{i j}\right)\right), \min \left(a_{i j}^{\prime}, b_{i j}^{\prime} c_{i j}^{\prime}\right)\right\rangle\right)$

$$
\begin{aligned}
& =\left(\left\langle\max \left(\max \left(a_{i j}, b_{i j}\right), \max \left(a_{i j}, c_{i j}\right)\right), \min \left(a_{i j}^{\prime}, b_{i j}^{\prime} c_{i j}^{\prime}\right)\right\rangle\right) \\
& \neq(A \vee B) X_{I}(A \vee C) .
\end{aligned}
$$

Hence, $A \vee\left(B X_{I} C\right) \neq(A \vee B) X_{I}(A \vee C)$. (By using Lemma 2.7)

The proof $(i i)$ is similar to that of $(i)$.

Property 5.8. Let $A, B$ and $C$ are any three IFMs of same size, we have

(i) $A \vee\left(B X_{2} C\right) \neq(A \vee B) X_{2}(A \vee C)$.

(ii) $A \wedge\left(B X_{2} C\right) \neq(A \wedge B) X_{2}(A \wedge C)$.

Proof: $(i) A \vee\left(B X_{2} C\right)=\left(\left\langle\max \left(a_{i j}, b_{i j} c_{i j}\right), \min \left(a_{i j}^{\prime}, \max \left(b_{i j}^{\prime}, c_{i j}^{\prime}\right)\right)\right\rangle\right)$

$$
\begin{aligned}
& =\left(\left\langle\max \left(a_{i j}, b_{i j} c_{i j}\right), \max \left(\min \left(a_{i j}^{\prime}, b_{i j}^{\prime}\right), \min \left(a_{i j}^{\prime}, c_{i j}^{\prime}\right)\right)\right\rangle\right) \\
& \neq(A \vee B) X_{2}(A \vee C) .
\end{aligned}
$$

Hence, $A \vee\left(B X_{2} C\right) \neq(A \vee B) X_{2}(A \vee C)$. (By using Lemma 2.7)

The proof $(i i)$ is similar to that of $(i)$.

Property 5.9. If $A$ and $B$ are any two IFMs of same size, we have

(i) $A X_{1} B \geq A \wedge B$.

(ii) $A X_{2} B \geq A \vee B$.

(iii) $A X_{2} B \geq A \wedge B$.

(iv) $A X_{1} B \neq A \vee B$.

Proof: $(i) A X_{I} B=\left(\left\langle\max \left(a_{i j}, b_{i j}\right), a_{i j}^{\prime} b_{i j}^{\prime}\right\rangle\right)$ and

$A \wedge B=\left(\left\langle\min \left(a_{i j}, b_{i j}\right), \max \left(a_{i j}^{\prime}, b_{i j}^{\prime}\right)\right\rangle\right)$.

Since $\max \left(a_{i j}, b_{i j}\right) \geq \min \left(a_{i j}, b_{i j}\right)$ and $a_{i j}^{\prime} b_{i j}^{\prime} \leq \max \left(a_{i j}^{\prime}, b_{i j}^{\prime}\right)$,

Hence, $A X_{1} B \geq A \wedge B$.

(ii) $A X_{2} B=\left(\left\langle a_{i j} b_{i j}, \max \left(a_{i j}^{\prime}, b_{i j}^{\prime}\right)\right\rangle\right)$ and

$A \vee B=\left(\left\langle\max \left(a_{i j}, b_{i j}\right), \min \left(a_{i j}^{\prime}, b_{i j}^{\prime}\right)\right\rangle\right)$.

Since $a_{i j} b_{i j} \leq \max \left(a_{i j}, b_{i j}\right)$ and $\max \left(a_{i j}^{\prime}, b_{i j}^{\prime}\right) \geq \min \left(a_{i j}^{\prime}, b_{i j}^{\prime}\right)$,

Hence, $A X_{2} B \geq A \vee B$.

(iii) Since $a_{i j} b_{i j} \leq \min \left(a_{i j}, b_{i j}\right)$ and $\max \left(a_{i j}^{\prime}, b_{i j}^{\prime}\right) \geq \min \left(a_{i j}^{\prime}, b_{i j}^{\prime}\right)$,

Hence, $A X_{2} B \geq A \wedge B$.

(iv) Since $a_{i j}^{\prime} b_{i j}^{\prime} \leq \min \left(a_{i j}^{\prime}, b_{i j}^{\prime}\right)$, for all $\mathrm{i}, \mathrm{j}$.

Hence, $A X_{1} B \neq A \vee B$.

Property 5.10. If $A$ and $B$ are any two IFMs of same size, we have

(i) $(A \vee B) \vee\left(A X_{1} B\right)=A X_{1} B$.

(ii) $(A \wedge B) \wedge\left(A X_{2} B\right)=A X_{2} B$.

Proof: $(i)(A \vee B) \vee\left(A X_{1} B\right)$

$$
=\left(\left\langle\max \left(\max \left(a_{i j}, b_{i j}\right), \max \left(a_{i j}, b_{i j}\right)\right), \min \left(\min \left(a_{i j}^{\prime}, b_{i j}^{\prime}\right), a_{i j}^{\prime} b_{i j}^{\prime}\right)\right\rangle\right)
$$


D.Venkatesan and S.Sriram

$$
\begin{aligned}
& =\left(\left\langle\max \left(a_{i j}, b_{i j}\right), a_{i j}^{\prime} b_{i j}^{\prime}\right\rangle\right) \\
& =A X_{1} B .
\end{aligned}
$$

Hence, $(A \vee B) \vee\left(A X_{1} B\right)=A X_{1} B$.

The proof $(i i)$ is similar to that of $(i)$.

Property 5.11. If $A$ and $B$ are any two IFMs of same size, we have

(i) $(A \wedge B) X_{1}(A \vee B)=A X_{1} B$.

(ii) $(A \wedge B) X_{2}(A \vee B)=A X_{2} B$.

Proof: $(i)(A \wedge B) X_{l}(A \vee B)$

$$
\begin{aligned}
& =\left(\left\langle\max \left(\min \left(a_{i j}, b_{i j}\right), \max \left(a_{i j}, b_{i j}\right)\right), \max \left(a_{i j}^{\prime}, b_{i j}^{\prime}\right) \min \left(a_{i j}^{\prime}, b_{i j}^{\prime}\right)\right\rangle\right) \\
& =\left(\left\langle\max \left(a_{i j}, b_{i j}\right), a_{i j}^{\prime} b_{i j}^{\prime}\right\rangle\right) \\
& =A X_{1} B .
\end{aligned}
$$

Hence, $(A \wedge B) X_{1}(A \vee B)=A X_{1} B$.

The proof $(i i)$ is similar to that of $(i)$.

\section{Conclusions}

The set of all IFMs with respect to the operations $X_{1}$ and $X_{2}$ form a commutative monoid. The operations $X_{1}$ and $X_{2}$ of IFMs are satisfy the De Morgan's laws. Distributive laws max-min and min-max compositions over $X_{1}$ and $X_{2}$ are proved and established some algebraic properties.

\section{REFERENCES}

1. A.K.Adak, M.Bhowmik and M.Pal, Intuitionistic fuzzy block matrix and its some properties, Annals of Pure and Applied Mathematics, 1(1) (2012) 13-31.

2. K.Atanassov, Intuitionistic fuzzy set, Fuzzy Sets and Systems, 20 (1986) 87-96.

3. K.Atanassov, E.Szmidt and J.Kacprzyk, Intuitionistic fuzzy implication $\rightarrow_{187}$, Notes on Intuitionistic Fuzzy Sets, 23(2) (2017) 37-43.

4. J.Boobalan and S.Sriram, Arithmetic operations on intuitionistic fuzzy matrices, Proceedings of the International Conference on Mathematical Sciences, Sathyabama University (2014), 484-487.

5. H.Bustince, Constraction of intuitionistic fuzzy relations with predetermined properties, Fuzzy Sets and Systems, 109 (2003) 379-404.

6. E.G.Emam and M.A.Fndh, Some results associated wiith the max-min and min-max compositions of bifuzzy matrices, Journal of the Egyption Mathematical Society, (2016) 515-521.

7. Y.B.Im, E.B.Lee and S.W.Park, The determinant of square intuitionistic fuzzy matrices, Far East Journal of Mathematical Sciences, 3(5) (2001) 789-796.

8. S.K.Khan, M.Pal and A.K.Shyamal, Intuitionistic fuzzy matrices, Notes on Intuitionistic Fuzzy Sets, 8(2) (2002) 51-62.

9. S.K.Khan and M.Pal, Some operations on intuitionistic fuzzy matrices, Acta Ciencia Indica, XXXIIM, (2006) 515-524.

10. K.H.Kim and R.W.Roush, Generalized fuzzy matrices, Fuzzy Sets and Systems, 4 (1980) 293-315.

11. H.Y.Lee and N.G.Jeong, Canonical form of a transitive intuitionistic fuzzy matrices, 


\section{Multiplicative Operations of Intuitionistic Fuzzy Matrices}

Honam Math. J., 27(4) (2005) 543-550.

12. A.R.Meenakshi and T.Gandhimathi, Intuitionistic fuzzy relational equations, Advances in Fuzzy Mathematics, 5(3) (2010) 239-244.

13. T.Muththuraji, S.Sriram and P.Murugadas, Decomposition of intuitionistic fuzzy matrices, Fuzzy Information and Engineering, 8 (2016) 345-354.

14. T.Muththuraji and S.Sriram, Some remarks on Lukasiwicz disjunction and conjunction operators on intuitionistic fuzzy matrices, Journal of advances in Mathematics, 11(3) (2015) 5000-5006.

15. M.Pal, Intuitionistic fuzzy determinant, V.U.J. Physical Sciences, 7 (2001) 87-93.

16. S.Sriram and P.Murugadas, On semi-ring of intuitionistic fuzzy matrices, Applied Mathematical Science, 4(23) (2010) 1099-1105.

17. S.Sriram and J.Boobalan, Monoids of intuitionistic fuzzy matrices, Annals of Fuzzy Mathematics and Informatics, 2 (3) (2016) 505-510.

18. A.K.Shyamal and M.Pal, Distances between intuitionistic fuzzy matrices, V.U.J. Physical Sciences, 8 (2002) 81-91.

19. A.K.Shyamal and M.Pal, Two new operators on fuzzy matrices, J. Appl. Math. \& Computing, 15 (2004) 91-107.

20. A.Trybulec and C.Bylinski, Some Properties of real numbers operations: min, max, square and square root, J. of Formalized Mathematics, 1 (1989) 1-4.

21. Z.Wang, Z.S.Xu, S.S.Liu and J.Tang, A netting clustering analysis method under intuitionistic fuzzy environment, Applied Soft Computing, 11(8) (2011) 5558-5564.

22. L.A.Zadeh, Fuzzy sets, Journal of Information and Control, 8 (1965) 338-353.

23. Y.Zhang and M.Zheng, New operators on fuzzy matrices, Fourth International workshop on Advanced computational Intelligence, Wu Han, China, (2011) 295-299.

24. M.Pal, Fuzzy matrices with fuzzy rows and columns, Journal of Intelligent and Fuzzy Systems, 30(1) (2016) 561-573.

25. M.Pal, Interval-valued fuzzy matrices with interval-valued fuzzy rows and columns, Fuzzy Information and Engineering, 7(3) (2015) 335-368. 